\title{
Observing Charge Transfer and Charge Defects by HREM Imaging
}

\author{
B. Deng and L. D. Marks
}

Department of Materials Science and Engineering, Northwestern University, Evanston, IL 60208

With the development of Cs-correctors to reach sub-angstrom resolution in electron microscopes, questions arise about the possibility of observing charge transfer and charge defects directly by HREM imaging. We simulated HREM images by multislice methods with structure factors from neutral atomic charge densities, ion charge densities, and self-consistent field (SCF) calculated crystal charge densities, denoted as I(neu), I(ion), and I(scf) respectively. Following this, the contrast due to charge transfer is evaluated by comparing the image difference between I(scf) and $\mathrm{I}(\mathrm{neu})$; the contrast due to charge defects is evaluated by comparing the image difference between I(scf) and I(ion). The imaging parameters are from an aberration-corrected Jeol2200FS microscope with a Cs value of $0.05 \mathrm{~mm}$. A constant temperature factor $\left(0.25 \AA^{2}\right)$ was used in all simulations.

The first case we studied was forsterite [001] HREM images [1]. Fig. 1(a) shows a typical difference image I(scf-neu), in which bright or dark spots are assigned to corresponding atomic columns (see Fig. 1(b)). Here we propose a simple method to interpret the image contrast due to charge transfer. When neutral atoms form the forsterite $\mathrm{Mg}_{2} \mathrm{SiO}_{4}$, the Coulomb potential in different columns may increase or decrease. Along column 1, all the Mg sites become more positive on charge transfer. For column 2, the electrons densities around $\mathrm{O}$ sites increase. Consequently the nuclear Coulomb potential is better screened. We also found that anions seem to lead to larger effects than cations. Column 3 with mixed ions displays same contrast as column 2. The contrast due to charge transfer contributes about $5-15 \%$ of overall contrast, depending on imaging parameters and zone axes. With current CCD detectors, these effects are in principle detectable.

As a representative model to observe charge defects in HREM imaging, we have used the reconstructed $\mathrm{MgO}(111)-\sqrt{3} \times \sqrt{3} R 30^{\circ}$ surface, denoted as $\mathrm{MgO}$ Rt3 for simplicity [2]. In the $\mathrm{Mg}$ terminated $\mathrm{MgO}$ Rt3 structure, two additional holes are required in the second $\mathrm{O}$ layer for charge neutrality. Fig. 2 shows its structure model, HREM image, and difference images. When referenced to neutral atoms, $\mathrm{Mg}$ and $\mathrm{O}$ layers display different contrast due to charge transfer in the difference image I(scf-neu) (Fig. 2(c)). Since MgO is close to an ideal ionic compound, neutral atoms are not particularly representative of the bulk structure factors. An alternative approach is to reference against ions, which should eliminate effects in the bulk and give a more local representation of the surface charge defects (Fig. 2(d)). The contrast due to charge defects is even larger than that due to charge transfer, contributing to about $20-30 \%$ of the overall contrast.

In summary, our simulations indicate that charge transfer and charge defects may be detectable directly by HREM imaging [3].

References

[1] B. Deng and L. D. Marks, Acta. Cryst. A, (2006) submitted.

[2] B. Deng, L. D. Marks, and J. M. Rondinelli, in preparation.

[3] This research was supported by the Department of Energy under grant No. DE-FG0201ER45945. The aid of Dr. Lan Yun Chang is acknowledged. 


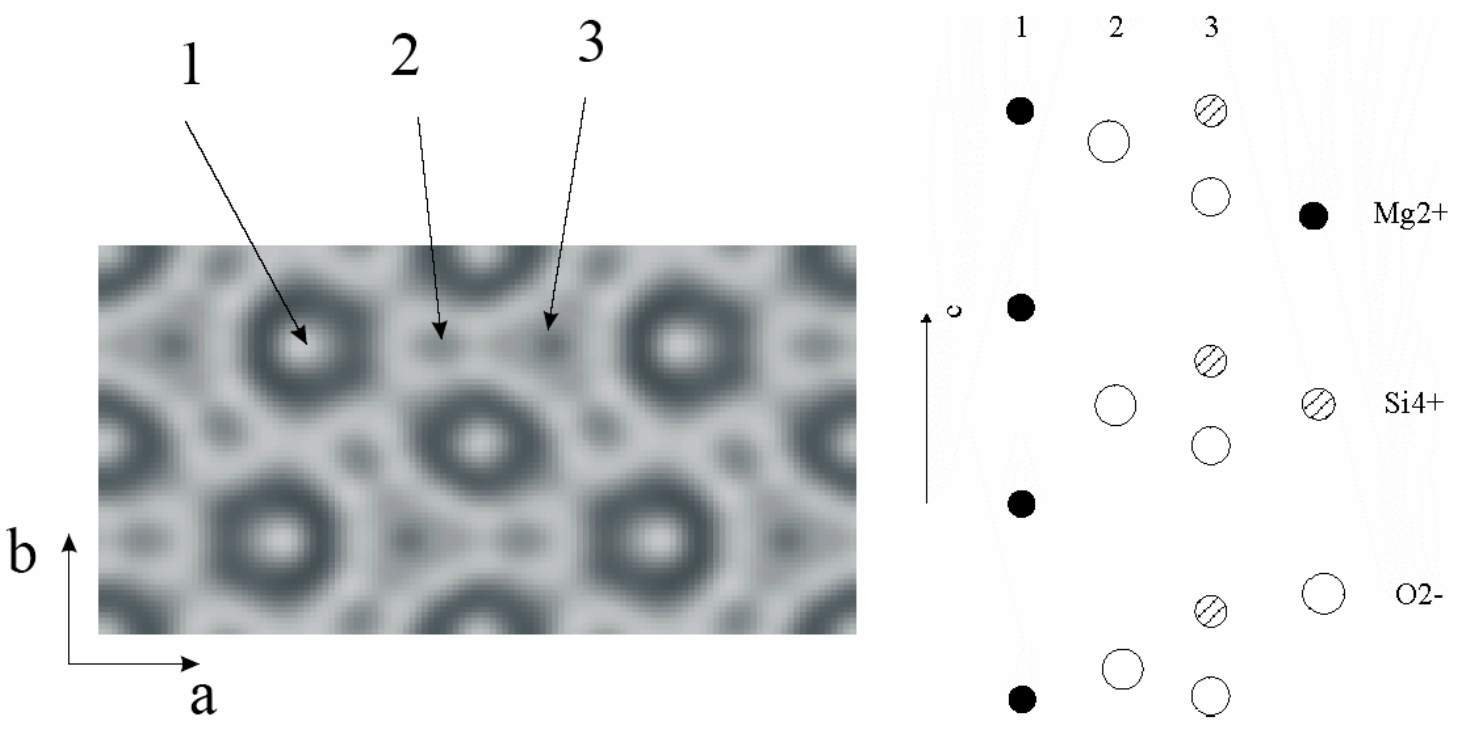

(a)

(b)

FIG. 1. Difference image I(scf-neu) for forsterite[001] (a) and its corresponding atomic columns (b).

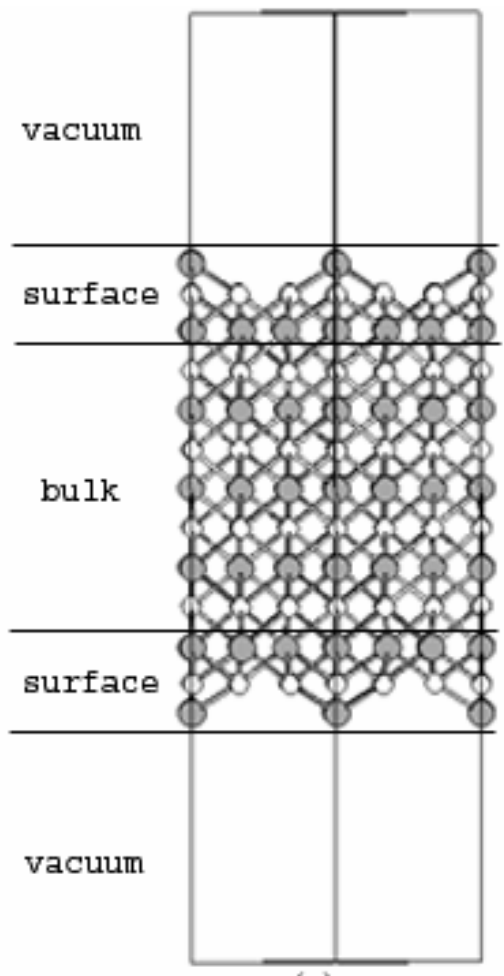

(a)

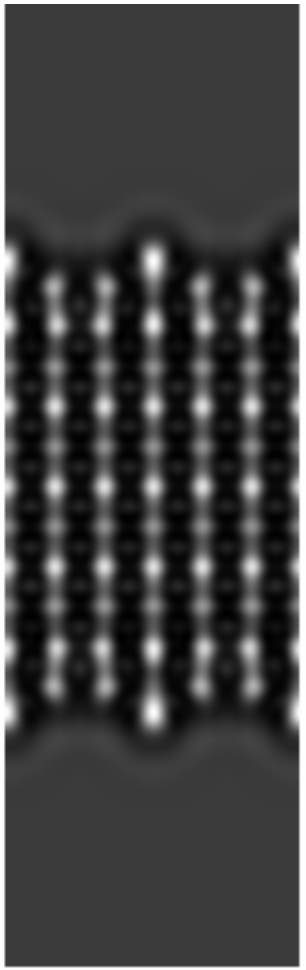

(b)

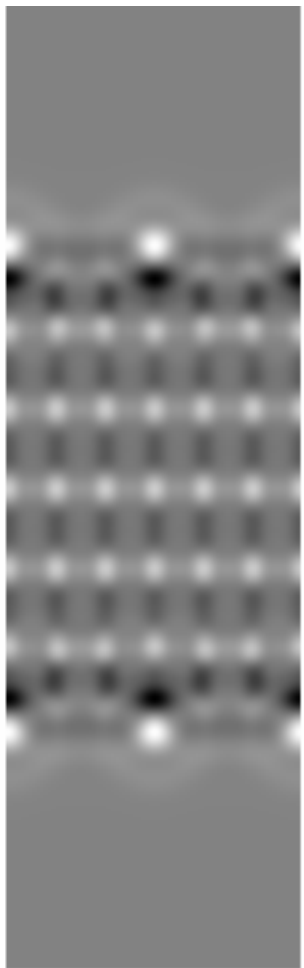

(c)

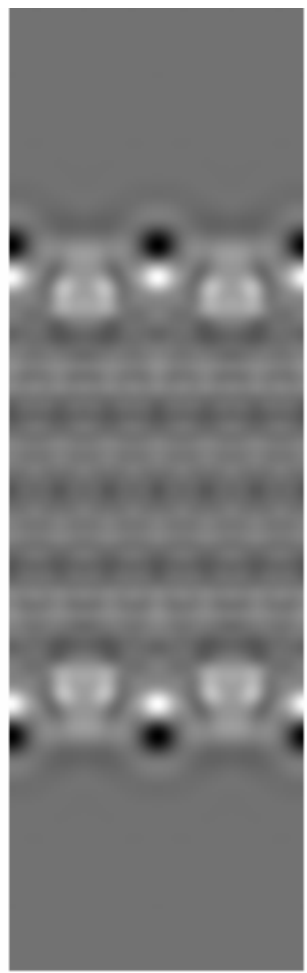

(d)

FIG. 2. The 13-layer structure of $\mathrm{MgO}(111) \mathrm{Rt3}$ viewed along [110] (a) and its image I(scf) (b), difference image I(scf-neu) (c), and difference image I(scf-ion). 